\title{
A Ramsey-style extension of a theorem of Erdös and Hajnal
}

\author{
by
}

Péter Komjáth (Budapest)

\begin{abstract}
If $n, t$ are natural numbers, $\mu$ is an infinite cardinal, $G$ is an $n$-chromatic graph of cardinality at most $\mu$, then there is a graph $X$ with $X \rightarrow(G)_{\mu}^{1},|X|=\mu^{+}$, such that every subgraph of $X$ of cardinality $<t$ is $n$-colorable.
\end{abstract}

The Erdős-Hajnal result we would like to generalize states that there are arbitrarily large chromatic graphs omitting short odd circuits ([1]). We reformulate this as follows. If $\mu$ is a cardinal, $t$ is a natural number, then there exists a graph $X$ with $X \rightarrow\left(K_{2}\right)_{\mu}^{1}$ such that every subgraph of $X$ on at most $t$ points is bipartite. Here $K_{2}$ is the one-edge graph and $X \rightarrow(Y)_{\mu}^{1}$ denotes that every vertex coloring of the graph $X$ with $\mu$ colors admits a monocolored (but not necessarily induced) copy of $Y$. If we take into consideration another result of Erdős and Hajnal (also in [1]), namely, that if $X \rightarrow\left(K_{2}\right)_{\omega}^{1}$ then $X$ contains every finite bipartite graph, we get the somewhat stronger statement that if $K_{t, t}$ is the complete bipartite graph with bipartition classes of size $t$, then there exists, for every cardinal $\mu$ and every finite $t$, a graph $X$ with $X \rightarrow\left(K_{t, t}\right)_{\mu}^{1}$ such that all subgraphs of $X$ on less than $t$ vertices are bipartite. This is the result we want to generalize; for any natural number $n$, we take an arbitrary $n$-chromatic graph in place of $K_{t, t}$ and we require that all subgraphs on less than $t$ vertices should be $n$-chromatic. For the proof, we somewhat modify the original Erdős-Hajnal construction (no surprise), we use another Specker-style graph.

Notation. We use the standard set theory notation. If $S$ is a set and $\mu$ a cardinal, then $[S]^{\mu}=\{X \subseteq S:|X|=\mu\}$. We write $\mathbf{x}=\left\{x_{1}, \ldots, x_{n}\right\}_{<}$ to denote that we enumerate the elements of $\mathrm{x}$ in increasing order.

2000 Mathematics Subject Classification: Primary 05C55, 03E05.

Research partially supported by the Hungarian National Science Research Grant No. T 019367. 
THEOREM. If $n, t$ are natural numbers, $\mu$ is an infinite cardinal, and $G$ is an n-chromatic graph of cardinality at most $\mu$, then there is a graph $X$ with $X \rightarrow(G)_{\mu}^{1},|X|=\mu^{+}$, such that every subgraph of $X$ of cardinality $<t$ is n-colorable.

Proof. Set $a=2 t, b=2 n t+1$. The ground set of the graph $X$ will be $\left[\mu^{+}\right]^{a b}$, the set of $a b$-element subsets of $\mu^{+}$. We join two elements $\mathbf{x}=$ $\left\{x_{1}, \ldots, x_{a b}\right\}<$ and $\mathbf{y}=\left\{y_{1}, \ldots, y_{a b}\right\}<$ if there is some $1 \leq k<n$ such that

$$
x_{k a}<x_{k a+1}<y_{1}<x_{k a+2}<y_{2}<\ldots<x_{a b}<y_{(b-k) a}
$$

or the other way round.

Claim 1. If $\mathbf{x}=\left\{x_{1}, \ldots, x_{a b}\right\}_{<}$and $\mathbf{y}=\left\{y_{1}, \ldots, y_{a b}\right\}_{<}$are joined and the interval $\left[x_{\alpha}, x_{\alpha+1}\right]$ of $\mathbf{x}$ intersects the interval $\left[y_{\beta}, y_{\beta+1}\right]$ of $\mathbf{y}$ then either $\alpha=\beta+k a$, or $\alpha=\beta+k a+1$, or the other way round.

Proof. Straightforward.

Claim 2. Every subgraph of $X$ with less than $t$ vertices can be $n$-colored.

Proof. Let $H$ be such a graph. We can assume that $H$ is connected (as otherwise we can split $H$ into connected components). Then the vertices of $H$ can be enumerated as $\left\{\mathbf{v}_{0}, \ldots, \mathbf{v}_{s}\right\}$ (for some $s$ ) so that every $\mathbf{v}_{i}$ with $i>0$ is joined to some $\mathbf{v}_{j}$ with $0 \leq j<i$. Set $\mathbf{v}_{i}=\left\{v_{1}^{i}, \ldots, v_{a b}^{i}\right\}_{<}$and define $\xi=v_{2 n t^{2}+t}^{0}$. For every $0 \leq i \leq s$ let $c(i)$ be the largest index for which $v_{c(i)}^{i} \leq \xi$. Clearly, $c(0)=2 n t^{2}+t$. We claim that there exist numbers $1 \leq d_{i} \leq 2 n t-1$ (for $\left.0 \leq i \leq s\right)$ such that

$$
a d_{i}+t-i \leq c(i) \leq a d_{i}+t+i .
$$

In fact, we show by induction on $i$ that there exists such a $d_{i}$ with $\left|d_{i}-n t\right| \leq$ $i n$. For $i=0, d_{0}=n t$ does the job. By induction, if we have the statement up to and including $i$, then using Claim 1 , we find that for some $0 \leq j \leq i$, $c(i+1)$ assumes the value $c(j)+k a$, or $c(j)+k a+1$, or $c(j)-k a$, or $c(j)-k a-1$ for some $1 \leq k<n$. Then we can choose $d_{i+1}=d_{j} \pm k$.

We now show that $f\left(\mathbf{v}_{i}\right)=d_{i}(\bmod n)$ is a good coloring of $H$. Assume that $f\left(\mathbf{v}_{i}\right)=f\left(\mathbf{v}_{j}\right)$ and they are joined. Again, by Claim 1, for some $1 \leq$ $k<n, c(i)=c(j)+k a$, or $c(i)=c(j)+k a+1$ (or vice versa). From this, we get $\left|a\left(d_{i}-d_{j}-k\right)\right| \leq 2 t-1$, so $d_{i}=d_{j}+k$, and so they do not get the same color.

Claim 3. $X \rightarrow(G)_{\mu}^{1}$.

Proof. Assume that we are given a coloring $F:\left[\mu^{+}\right]^{a b} \rightarrow \mu$ of the ground set. We are going to show that for some color $\tau<\mu$ the complete $n$-partite graph $K_{\mu, \ldots, \mu}$, with color classes of cardinality $\mu$, is embeddable into the subgraph of those vertices which get color $\tau$. 
We define the formulas $\varphi_{0}^{\tau}, \varphi_{1}^{\tau}\left(x_{1}\right), \ldots, \varphi_{i}^{\tau}\left(x_{1}, \ldots, x_{i}\right)$ for all $i \leq a b$ as follows. $\varphi_{a b}^{\tau}\left(x_{1}, \ldots, x_{a b}\right)$ denotes that $x_{1}<\ldots<x_{a b}$ and $F\left(x_{1}, \ldots, x_{a b}\right)=\tau$. If $\varphi_{i+1}^{\tau}$ is defined, we let $\varphi_{i}^{\tau}\left(x_{1}, \ldots, x_{i}\right)$ stand for

$$
\exists^{*} y \varphi_{i+1}^{\tau}\left(x_{1}, \ldots, x_{i}, y\right)
$$

where the quantifier $\exists^{*} y$ reads "there exist $\mu^{+}$elements $y$ such that ...".

SubClaim. There is a $\tau<\mu$ such that $\varphi_{0}^{\tau}$ holds.

Proof. Assume, for a contradiction, that $\varphi_{0}^{\tau}$ is false for every $\tau<\mu$. Spelled out, this means that, for every $\tau<\mu$ there is a bound $\alpha_{\tau}$ such that $\varphi_{1}^{\tau}\left(x_{1}\right)$ is false for $x>\alpha_{\tau}$. There is a common bound for all these bounds, so there is an $x_{1}$ for which every $\varphi_{1}^{\tau}\left(x_{1}\right)$ is false. Fix such an $x_{1}$ and proceed. We can find an $x_{2}>x_{1}$ such that $\varphi_{2}^{\tau}\left(x_{1}, x_{2}\right)$ is false for every $\tau<\mu$. Fix such an $x_{2}$, and continue. Eventually we get a sequence $\left\{x_{1}, \ldots, x_{a b}\right\}<$ such that $F\left(x_{1}, \ldots, x_{a b}\right)=\tau$ is false for every $\tau<\mu$, which is clearly impossible.

For the rest of the proof fix $\tau<\mu$ as in the Subclaim. Using induction we can find an increasing sequence of ordinals $0=\delta_{0}<\delta_{1}<\ldots<\delta_{r}(r<\omega)$ such that if $i<a b, r<\omega, x_{1}<\ldots<x_{i}<\delta_{r}$, and $\varphi_{i}^{\tau}\left(x_{1}, \ldots, x_{i}\right)$ holds then there are $\mu$ ordinals $\delta_{r}<y<\delta_{r+1}$ such that $\varphi_{i+1}^{\tau}\left(x_{1}, \ldots, x_{i}, y\right)$ is true. That is, there are $\mu$ elements $x_{1}<\delta_{1}$ for which $\varphi_{1}^{\tau}\left(x_{1}\right)$ holds, for each such $x_{1}$ there are $\mu$ elements $\delta_{1}<x_{2}<\delta_{1}$ for which $\varphi_{2}^{\tau}\left(x_{1}, x_{2}\right)$ holds, and so forth. (In fact, we could easily continue for $\mu^{+}$steps, but we will not need it.)

For $1 \leq i \leq n, 1 \leq j \leq b, 1 \leq m \leq a$ we let

$$
A_{j m}^{i}=\left[\delta_{r}, \delta_{r+1}\right)
$$

where

$$
r=(i+j)(n+1)(a+1)+m(n+1)+i,
$$

i.e., the pairwise disjoint sets $A_{j m}^{i}$ are ordered by $(i+j)$ first, then according to the value of $m$, and finally by $i$. In this order, we select from each of these sets $\mu$ elements: $x_{\alpha}^{i j m} \in A_{j m}^{i}(\alpha<\mu)$ in such a way that

$$
\varphi_{(j-1) a+m}^{\tau}\left(x_{\alpha}^{i 11}, x_{\alpha}^{i 12}, \ldots, x_{\alpha}^{i 1 a}, x_{\alpha}^{i 21}, \ldots, x_{\alpha}^{i 2 a}, \ldots, x_{\alpha}^{i j 1} \ldots, x_{\alpha}^{i j m}\right)
$$

holds. This can be done by the selection of the $\delta$ 's and the way the $A_{j k}^{i}$ 's were ordered. Finally, put $\mathbf{x}_{\alpha}^{i}=\left\{x_{\alpha}^{i j m}: 1 \leq j \leq b, 1 \leq m \leq a\right\}$.

Now consider $1 \leq i_{0}<i_{1} \leq n$, say $i_{1}=i_{0}+k$. The relative order of the sets in the families $\left\{A_{j m}^{i_{0}}: 1 \leq j \leq b, 1 \leq m \leq a\right\}$ and $\left\{A_{j m}^{i_{1}}: 1 \leq j \leq\right.$ $b, 1 \leq m \leq a\}$ will be the following (we replace superscript $i_{0}$ by a prime and superscript $i_{1}$ by two primes):

$$
\begin{aligned}
A_{11}^{\prime}<A_{12}^{\prime}<\ldots<A_{1 a}^{\prime}<A_{21}^{\prime}<\ldots<A_{k+1,1}^{\prime} & \\
& <A_{11}^{\prime \prime}<A_{k+1,2}^{\prime}<A_{12}^{\prime \prime}<\ldots,
\end{aligned}
$$


i.e., they are in one of the interlacing positions we defined connectivity in $X$. From this we conclude that if $1 \leq i_{0}<i_{1} \leq n, \alpha, \beta<\mu$, then $\mathbf{x}_{\alpha}^{i_{0}}$ and $\mathbf{x}_{\beta}^{i_{1}}$ are joined, so the vertices $\left\{\mathbf{x}_{\alpha}^{i}: 1 \leq i \leq n, \alpha<\mu\right\}$ give a $K_{\mu, \ldots, \mu}$ in color $\tau$.

\section{References}

[1] P. Erdős and A. Hajnal, On chromatic number of graphs and set systems, Acta Math. Acad. Sci. Hungar. 17 (1966), 61-99.

Department of Computer Science

Eötvös University

Kecskeméti u. 10-12

1053 Budapest, Hungary

E-mail: kope@cs.elte.hu 